\title{
Measurements of LET Distribution and Dose Equivalent onboard the Space Shuttle IML-2 (STS-65) and S/MM\#4 (STS-79)
}

Takayoshi Hayashi ${ }^{1}$, Tadayoshi Doke ${ }^{1}$, Jun Kikuchi ${ }^{1}$, Takao Sakaguchi ${ }^{1}$, Ryuichi Takeuchi ${ }^{1}$, Takeshi Takashima ${ }^{1}$, Masanori Kobayashi' ${ }^{1}$, Kazuhiro Terasawa ${ }^{1}$, Kenichi Takahashi' ${ }^{1}$, AkiraWatanabe ${ }^{1}$, Atsushi Kyan ${ }^{1}$, Nobuyuki Hasebe $^{2}$, Toshisuke Kashiwagi ${ }^{3}$, Koichi Ogura $^{4}$, Shunji Nagaoka ${ }^{5}$, Mitsuyasu Kato ${ }^{5}$, Tamotsu Nakano ${ }^{5}$, Shimpei Takahashi $^{5}$, Hisashi Yamanaka ${ }^{6}$, Katsumi Yamaguchi ${ }^{6}$ and Gautam D. Badhwar ${ }^{7}$

${ }^{1}$ Advanced Research Center for Science and Engineering, Waseda University, Okubo 3-4-1, Shinjuku-ku, Tokyo 169, Japan;

${ }^{2}$ Department of Computer Science, Ehime University, Bunkyo-cho 3, Matsuyama, Ehime 790, Japan;

${ }^{3}$ Department of Business and Engineering, Kanagawa University, Rokkakubashi 3-27-1, Kanagawa-ku, Yokohama 221, Japan;

${ }^{4}$ College of Industrial Technology, Nihon University, Izumi-cho 1-2-1, Narashino, Chiba 275, Japan;

${ }^{5}$ Tsukuba Space Center, NASDA, Sengen 2-1-1, Tsukuba, Ibaraki 305, Japan;

${ }^{6} J a p a n$ Space Utilization Promotion Center, Nishiwaseda 3-30-16, Shinjuku-ku, Tokyo 169, Japan; and

${ }^{7}$ NASA Johnson Space Center, Houston, TX 77058, U.S.A.

\begin{abstract}
Space radiation dosimetry measurements have been made onboard the Space Shuttle STS-65 in the Second International Microgravity Laboratory (IML-2: $28.5^{\circ}$ x $300 \mathrm{~km}: 14.68$ days) and the STS-79 in the 4th Shuttle MIR mission (S/MM\#4: $51.6^{\circ}$ x 300-400 km: 10.2 days). In these measurements, three kinds of detectors were used; one is a newly developed active detector telescope called "Real-time Radiation Monitoring Device (RRMD-I for IML-2 and RRMD-II with improved triggering system for S/MM\#4)" utilizing silicon semi-conductor detectors and the other detectors are conventional passive detectors of thermoluminescence dosimeters (TLDs) and CR-39 plastic track detectors. The main contribution to dose equivalent for particles with LET $>5.0 \mathrm{keV} / \mu \mathrm{m}(\mathrm{IML}-2)$ and LET $>3.5 . \mathrm{keV} / \mu \mathrm{m}(\mathrm{S} / \mathrm{MM \# 4})$ is seen to be due to galactic cosmic rays (GCRs) and the contribution of the South Atlantic Anomaly (SAA) is less than 5\%(IML2: $\left.28.5^{\circ} \times 300 \mathrm{~km}\right)$ and $15 \%\left(\mathrm{~S} / \mathrm{MM} \# 4: 51.6^{\circ} \times 400 \mathrm{~km}\right)$ in the above RRMD LET detection conditions. For the whole LET range $(>0.2 \mathrm{keV} / \mu \mathrm{m}$ ) obtained by TLDs and CR-39 in these two typical orbits (a small inclination $\mathrm{x}$ low altitude and a large inclination $\mathrm{x}$ high altitude), absorbed dose rates range from 94 to 114 $\mu \mathrm{Gy} /$ day, dose equivalent rates from 186 to $207 \mu \mathrm{Sv} /$ day and average quality factors from 1.82 to 2.00 depending on the locations and directions of detectors inside the Spacelab at the highly protected IML-2 orbit $\left(28.5^{\circ} \times 300 \mathrm{~km}\right)$, and also, absorbed dose rates range from 290 to $367 \mu \mathrm{Gy} / \mathrm{day}$, dose equivalent rates from 582 to $651 \mu \mathrm{Sv} /$ day and average quality factors from 1.78 to 2.01 depending on the dosimeter packages around the RRMD-II "Detector Unit" at the S/MM\#4 orbit $\left(51.6^{\circ} \times 400 \mathrm{~km}\right)$. In general, it is seen that absorbed doses depend on the orbit altitude (SAA trapped particles contribution dominant) and dose equivalents on the orbit inclination (GCR contribution dominant). The LET distributions obtained by two different types of active and passive detectors, RRMDs and CR-39, are in good agreement for LET of $15-200 \mathrm{keV} /$ $\mu \mathrm{m}$ and difference of these distributions in the regions of LET $<15 \mathrm{keV} / \mu \mathrm{m}$ and LET $>200 \mathrm{keV} / \mu \mathrm{m}$ can be explained by considering characteristics of CR-39 etched track formation especially for the low LET tracks and chemical etching conditions.
\end{abstract}

\section{Introduction}

Recently, several long-duration manned space missions have been scheduled. On such space missions, radiation environments are governed mainly by galactic cosmic ray particles (GCRs), solar flare particles and protons trapped in the Earth's radiation belts. To evaluate the radiation effects correctly in such a mixed field, accurate measure-

Recieved April 28, 1997

Accepted May 14, 1997

*Address correspondence to: Takayoshi Hayashi, Advanced

Research Center for Science and Engineering, Waseda

University, Okubo 3-4-1, Shinjuku-ku, Tokyo 169, Japan

Phone: 81-3-3203-4141 ext. 75-2125

Fax: 81-3-3203-3231

E-mail: ments of radiation must be made in a wide range of particle species from protons to iron and energies of $\sim \mathrm{MeV}$ to relativistic $(>\mathrm{GeV})$. Furthermore, information of the time and orbital location of spacecraft, that is, "real-time" radiation monitoring would be required for a long duration journey outside the geomagnetosphere to warn urgent radiation hazards such as big solar flare events.

The degree of radiation risk to human tissues, which is termed as "dose equivalent", is evaluated as the absorbed dose multiplied by a biological dose parameter such as a "quality factor" $Q . Q$ is a function of the linear energy transfer (LET) (ICRP, 1977; ICRP, 1991). As such, the measurement of the LET distribution is absolutely needed to obtain accurate dose equivalents at least in the region of 
LET $>3.5 \mathrm{keV} / \mu \mathrm{m}$ (ICRP, 1977) or LET $>10 \mathrm{keV} / \mu \mathrm{m}$ (ICRP, 1991) coupled with quality factor $Q$. For this purpose, a combination of different types of detectors would be useful. In the present monitoring, we use three types of detectors; one is a newly developed active detector telescopes, which allows the measurement of the LET distribution and radiation dosimetry "in real time" for LET $>5 \mathrm{keV} / \mu \mathrm{m}$ of RRMD-I (IML-2: Doke et al., 1995a) and for LET $>3.5 \mathrm{keV} / \mu \mathrm{m}$ of RRMD-II (S/MM\#4: Sakaguchi et al., 1997) at the detection discriminations and noise levels. The other detectors are conventional passive detectors, TLDs and CR-39 track detectors. TLDs can measure the total absorbed dose during flight for all values of LET $>0.2 \mathrm{keV} / \mu \mathrm{m}$, although they cannot give the LET distribution. CR-39 can be used to measure the LET distribution for LET $>3.9 \mathrm{keV} / \mu \mathrm{m}$, which is the detection threshold of the present CR-39 (Doke et al., 1995b; Benton et al., 1986).

From the above point of view, measurements of radiation dosimetry have been used to investigate biological effects of space radiation in the Life Science program of National Space Development Agency of Japan (NASDA) since STS-42 (IML-1) and STS-47 (SL-J) (Nagaoka et al., 1993). As a series of this program, we achieved the truly "real-time" radiation monitorings inside Spacelab (STS-65; $28.5^{\circ} \times 300 \mathrm{~km}$ ) called the Second International Microgravity Laboratory (IML-2) launched on 8 July, 1994, with a duration of 14.68 days and Spacehab (STS-79; $51.6^{\circ}$ x 300-400 km) called the 4th Shuttle MIR mission (S/ MM\#4) launched on 16 September, 1996, with a duration of 10.2 days. In this paper, we present the results of realtime measurements of LET distributions and radiation dosimetry by RRMD-I and RRMD-II, and an evaluation of absorbed doses, dose equivalents and average quality factors measured by TLDs and CR-39. The differences of contribution to dose equivalent by the Shuttle orbital parameters, the inclination and the altitude, are discussed. Also, a comparison of different types of active and passive detectors, RRMDs and CR-39, LET distributions during these missions is discussed.

\section{Instrumentation \\ 2.1. RRMDs}

So far, several dosimetries by active detectors such as tissue equivalent proportional counter (TEPC) have been achieved for several orbital parameters (Badhwar et al., 1992; 1994; 1996; Golightly et al., 1994). However, the results of these measurements have been evaluated only after the flight or needed large corrections with significant deconvolutions even in real time measurements. So, in order to monitor more accurately in real time, we developed a new device RRMD-I as mentioned above. Figure 1(a) shows a cross section of RRMD-I of eight layer silicon detectors with two position detectors PSD-1 and PSD-2 $\left(62 \times 62 \mathrm{~mm}^{2}\right)$ of a geometric factor $45.25 \mathrm{~cm}^{2} \mathrm{sr}$ for the IML-2 mission (Doke, 1994a; Hayashi et al., 1994; Doke et al., 1995a). The RRMD-I telescope is essentially (a)

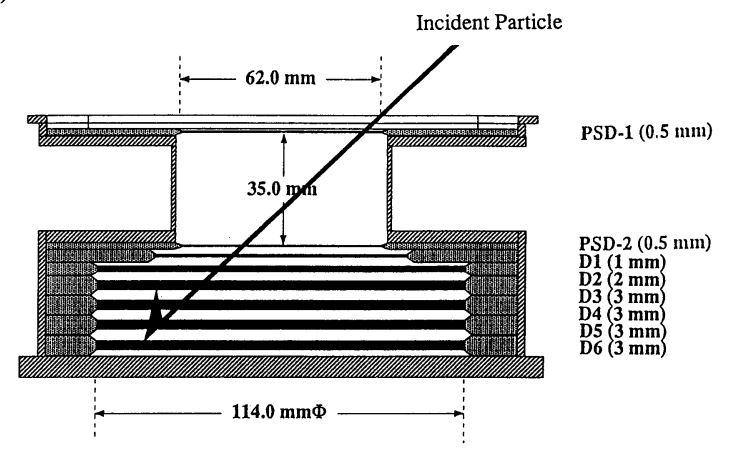

(b)

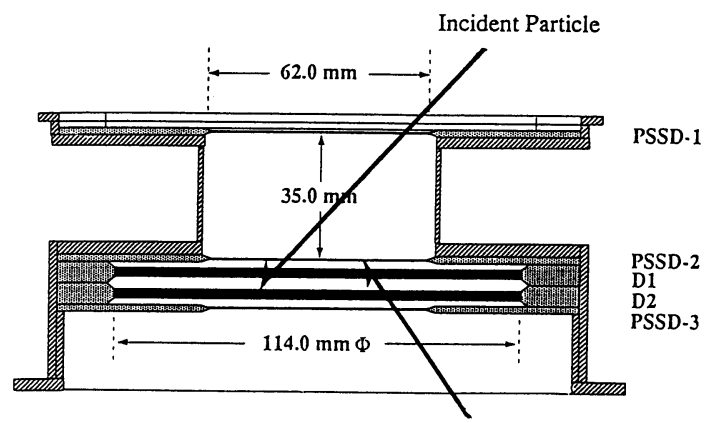

Fig. 1 (a) A schematic cross section of a multi-layer silicon telescope called Real time Radiation Monitoring Device (RRMD-I). Two position detectors (PSD-1 and PSD-2) are placed at the top to measure the incident angle of a particle. (b) A schematic cross section of a improved telescope RRMD-II with the third position detector(PSSD-3) at the bottom.

identical to the HI-telescope in the GEOTAIL mission (launched in July 1992) for study of heavy cosmic ray isotopes (Doke et al., 1994). In this telescope, the discrimination level of the triggering system using PSD-1 and PSD-2 has been set to detect only particles heavier than carbon. So, RRMD-I has been limited to detect only particles with LET $>5 \mathrm{keV} / \mu \mathrm{m}$ also due to the noise level. In addition, the RRMD-I contains only two position detectors which are used as the coincidence trigger detectors, where PSD-2 is defined as a standard dosimetry detector. Then, particles which stopped in PSD-2 from the bottom side entrance were not triggered. Therefore, an improved telescope RRMD-II was fabricated adding one more position detector (PSSD-3) at the bottom as shown in Fig. 1(b) with three position detectors (PSSD-1, PSSD-2 and PSSD3) of a geometric factor $87.04 \mathrm{~cm}^{2}$ sr for the S/ MM\#4 mission (Sakaguchi et al., 1997). Also, the discrimination level was improved to be LET $>3.5 \mathrm{keV} /$ $\mu \mathrm{m}$.

The RRMD-I instrument was installed at a corner of "Detector Unit" (Fig. 2(a)), whose position and direction inside Spacelab were changed approximately every two days to investigate possible spatial and directional 
(a)

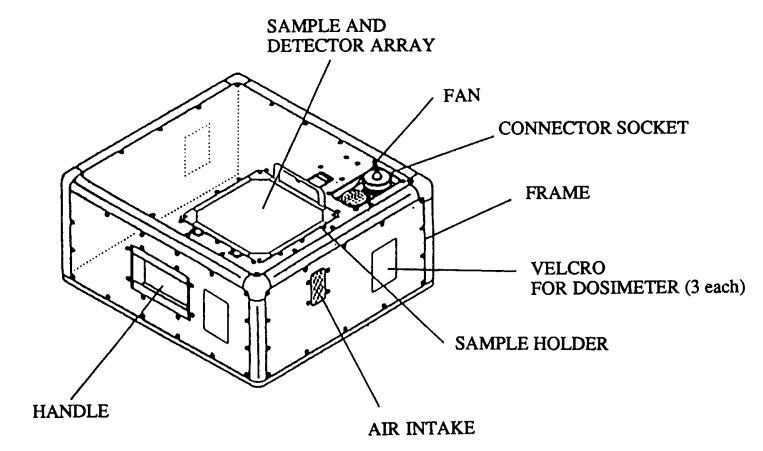

(b)
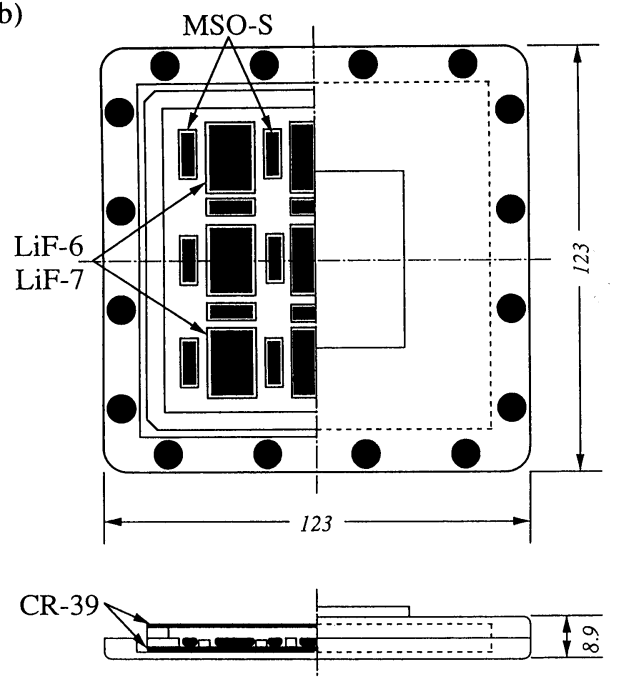

unit: $m m$

Fig. 2 (a) "Detector Unit" containing RRMD. (b) A schematic top and side views of the dosimeter package containing TLDs, CR-39 and biological samples. LiF-TLDs were not used in the S/ MM\#4 experiment.

(a)

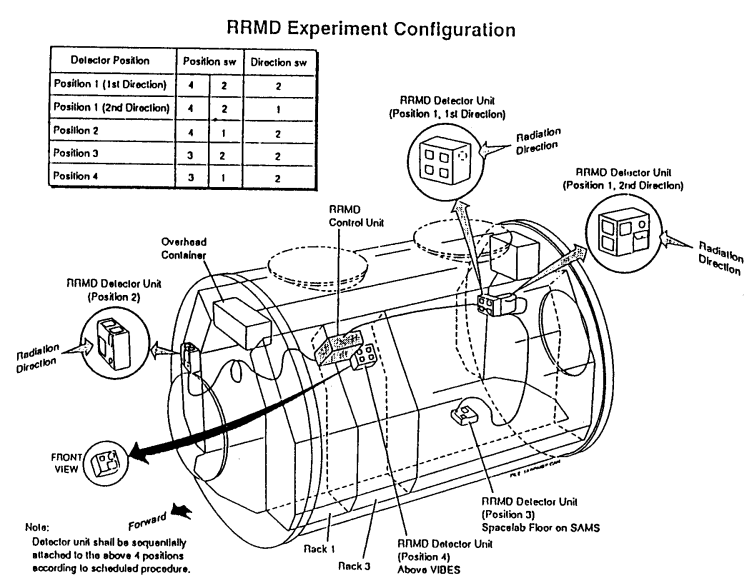

variations of space radiation in the case of the IML-2 as shown in Fig. 3(a) (Doke et al., 1995a). The RRMD-II was installed in the same "Detector Unit" and was placed on the ceiling of Spacehab (S/MM\#4) with the thinnest wall (Fig 3.(b)). Detailed description of in-flight calibration, real time dosimetry operation of RRMDs and corrections (less than $2 \%$ for dose equivalents) for particles stopped in the detector PSSD-2 of RRMD-II have been presented by Doke et al. (1995a) and Sakaguchi et al. (1997).

\subsection{TLDs and CR-39 plastic track detectors}

Two CR-39 plates $\left(125.0 \times 125.0 \times 1.2 \mathrm{~mm}^{3}\right)$ doped with antioxidant (Naugard 445) supplied by Fukuvi Chemical Industry which is named "HARZLAS" (Benton et al., 1986) were set in the top and bottom sides of "Sample Holder" (Fig. 2(a)), which includes the biological samples placed at the top of "Detector Unit" to check the radiation effects, and to compare LET distributions by RRMD and CR-39 in both experiments of IML-2 and S/MM\#4. In the IML-2 experiment, two dosimeter packages (F2 and F3) (Fig. 2(b)), which contain CR-39 (99.5 x $\left.99.5 \times 1.0 \mathrm{~mm}^{3}\right)$ and TLDs ( $\mathrm{LiF}$ and $\mathrm{Mg}_{2} \mathrm{SiO}_{4}$ (Tb)-MSO) supplied by Kasei Optonics Co., which also include biological samples, were attached to the side wall of "Detector Unit" and two other dosimeter packages (FI and F4) were placed in the incubator of the "BIORACK" and in the rack of the "PEMBSIS" to obtain the radiation data in the IML-2 experiment. In the S/MM\#4 experiment, all four dosimeters (Dosimeter 1-4) which are the same configuration as IML-2 ones with the exception of no use of LiF-TLD were set around the "Detector Unit".

Only MSO-TLD was used for further analysis because the sensitivity of LiF used in the IML-2 experiment was not stable both for flight units and ground control units. The MSO-TLD was calibrated with $\gamma$-rays of ${ }^{60} \mathrm{Co}$ and also with relativistic $550 \mathrm{MeV} / \mathrm{n}$ Fe and $920 \mathrm{MeV} / \mathrm{n} \mathrm{U}$ ions. In the calibration with heavy ions, an efficiency for absorbed dose obtained by MSO-TLD was found to be

(b)

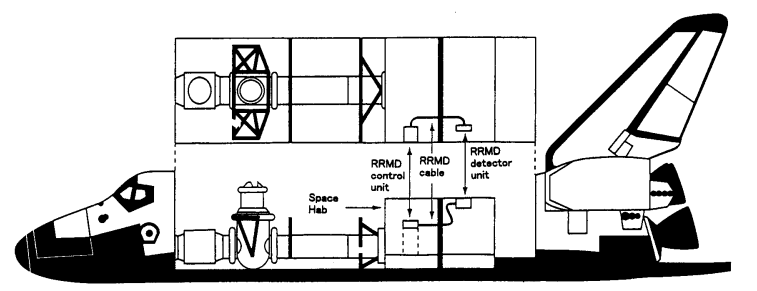

Fig. 3 (a) Positions and directions of "Detector Unit" for observation in the Spacelab (IML-2) . (b) Position and direction of "Detector Unit" for observation in the Spacehab (S/MM\#4) 
about $60 \%$ of calculated one for those high LET particles. This experimental fact allowed us to estimate dose equivalent for the whole LET range with a combination of data of TLDs and CR-39 (Doke et al., 1995b; Hayashi et al., 1996), which can measure the LET distribution for LET $>3.9 \mathrm{keV} / \mu \mathrm{m}$ in whose region quality factor $Q$ is a function of LET (ICRP, 1977; ICRP, 1991).

The CR-39 plates were calibrated using $20 \mathrm{MeV}$ protons and $25 \mathrm{MeV} / \mathrm{n}$ He ions at the TIARA Cyclotron of JAERI, Takasaki and heavy ions (135 MeV/n C, N and $\mathrm{Ne}$, and $88 \mathrm{MeV} / \mathrm{n} \mathrm{Ar}$ ) from the Ring Cyclotron of RIKEN. The chemical etching for CR-39 was carried out in $7 \mathrm{~N}$ $\mathrm{NaOH}$ solution at $70^{\circ} \mathrm{C}$ for $30 \mathrm{~h}$ for IML-2 and $27 \mathrm{~h}$ for $\mathrm{S} /$ MM\#4. Then, the relation between the normalized track etch rate and $\mathrm{REL}_{200}$ (Benton, 1968) was obtained (Hayashi et al., 1996), where track etch rate was obtained by measuring major and minor axes of the surface ellipse of a etched track and a removed surface material, and $\mathrm{REL}_{200}$ was simply converted to LET as LET $(\mathrm{keV} / \mu \mathrm{m}$-water $)=$ $0.19 \times \mathrm{REL}_{200}\left(\mathrm{MeV} \mathrm{cm}^{2} / \mathrm{g}-\mathrm{CR}-39\right)$ using the ratio of the stopping powers in water to $\mathrm{REL}_{200}$ in CR-39 for near relativistic particles. Although there is some weak variation of a value 0.19 for the very low energy region, the effect of such a small variation is negligible at the present dosimetry measurements.

(a)

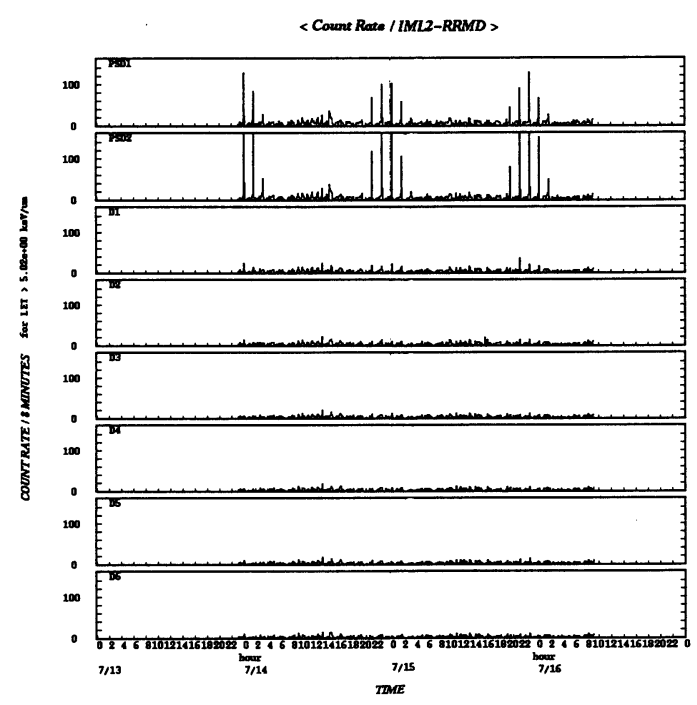

\section{Results}

\subsection{RRMD-I Results (IML-2)}

The RRMD-I instrument has been designed to measure LET values of incident particles event by event in individual eight silicon detectors (Fig. 1(a)), to construct LET distribution and to estimate absorbed dose and dose equivalent rates "in real time" during the flight as mentioned above. Using the triggering system by the coincidence of two detectors, PSD-1 and PSD-2, the contribution of particles entering from the bottom and stopping in PSD-2 is neglected for the dosimetry analysis at a standard dosimetry detector PSD-2. However, such a contribution to dose equivalent was experimentally determined to be insignificant in the flight of low inclination $\left(28.5^{\circ}\right)$ and the low altitude $(300 \mathrm{~km})$ flight with smaller contribution (less than $2 \%$ for LET $>5.0 \mathrm{keV} / \mu \mathrm{m}$ ) of trapped protons and low energy GCRs (Doke et al., 1995a). This small value of contribution was confirmed to be about $1.3 \%$ by the $\mathrm{S} /$ MM\#4 experiment for particles with LET $>3.5 \mathrm{keV} / \mu \mathrm{m}$ (Sakaguchi et al., 1977).

Figure 4(a) shows an example of the temporal variation of counting rate of particles with LET $>5 \mathrm{keV} / \mu \mathrm{m}$ obtained from individual eight silicon detectors (PSD-1-D-6). In this figure, clear peaks in PSD-1 and PSD-2 are seen with a time interval of about 90 min corresponding to the passage

(b)

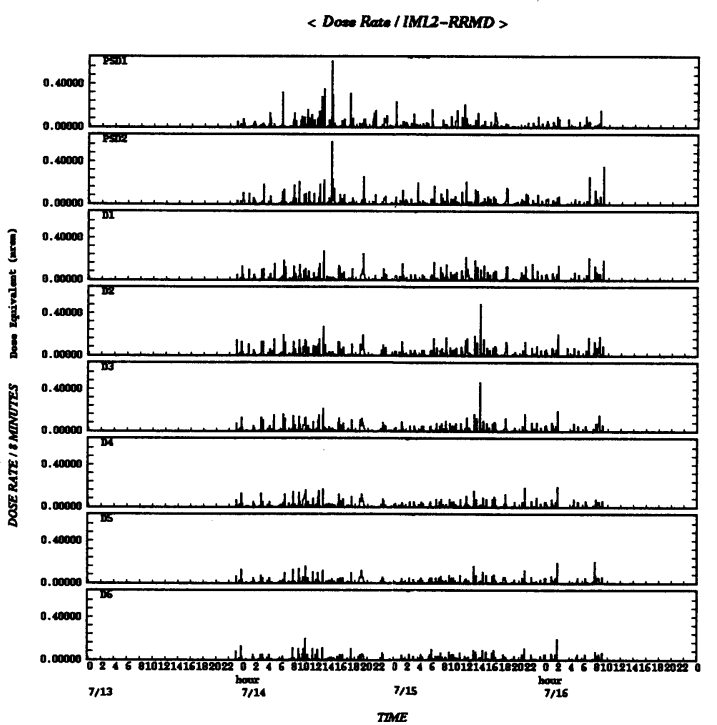

Fig. 4 (a) An example of temporal variation of counting rate of particles of LET $>5 \mathrm{keV} / \mu \mathrm{m}$ (IML-2)

(b) An example of temporal variation of dose equivalent rate of particles of LET $>5 \mathrm{keV} / \mu \mathrm{m}$ (IML-2). 
of the Shuttle through the SAA. These particles were assigned to be mainly trapped protons of $\sim 10 \mathrm{MeV}$ at the detector position by the spectroscopy of energy loss and total energy in multi-layer silicon detectors. Figure 4(b) also shows an example of the temporal variation of dose equivalent rate by particles with LET $>5 \mathrm{keV} / \mu \mathrm{m}$, which was calculated by the integration of LET distributions multiplied by quality factors $Q$ using the solid angle of the RRMD telescope (Doke et al., 1995b; Hayashi et al., 1996). As seen in this figure, the contribution to dose equivalent from trapped protons at the SAA is not very significant for LET $>5 \mathrm{keV} / \mu \mathrm{m}$, while $12 \mathrm{~h}$ periodic small enhancements of dose equivalent rate are seen at the orbital position of the Shuttle corresponding to the lower cut-off rigidity region of geomagnetic field above Mexico and Australia, where the particle density of lower energy and high LET GCRs is considered to be increased (Hayashi et al., 1996).

The same kind of situation is also seen in world-wide maps (Figs. 5(a), (b) and (c)). Figure 5(a) shows the Shuttle positions every two minutes in which the particle density for LET $>5 \mathrm{keV} / \mu \mathrm{m}$ is greater than 5 particles $/ \mathrm{min}$ in PSD2. In this figure, the cluster in the northern part of the SAA by trapped protons can clearly be seen. In Fig. 5(b), on the other hand, the positions of incidence of high LET particles of LET $>100 \mathrm{keV} / \mu \mathrm{m}$ seem to be randomly scattered showing weakly clusterized regions at larger latitudes, again

(a)

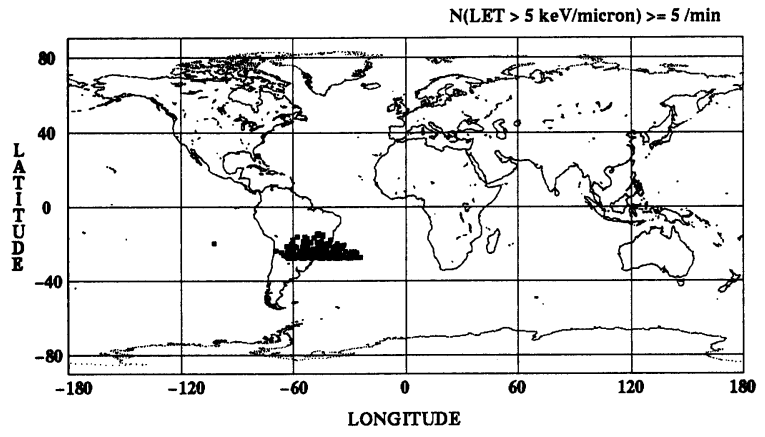

especially above Mexico and Australia. Also, the same pattern can be seen in Fig. 5(c) for the positions of high dose equivalent rate $(>0.5 \mu \mathrm{Sv} / \mathrm{min})$. Thus, most of contribution to total dose equivalent by particles of LET $>$ $5 \mathrm{keV} / \mu \mathrm{m}$ is understood to be governed by high energy GCRs in the STS-65 flight with a small inclination $\left(28.5^{\circ}\right.$ ) and a low altitude $(300 \mathrm{~km})$ mission. Actually, the contribution of the SAA region was less than $5 \%$ to the total dose equivalent. (Note that the data telemeter at the region of $-25^{\circ}$ to $80^{\circ}$ longitude has not fully been covered as $100 \%$ efficiency because of shadows in satellite communications.)

Figures 6(a) and (b) show examples of differential LET distributions for trapped particles(a) and $\operatorname{GCRs}(b)$, respectively, observed in eight silicon detectors. In,Fig. 6(a) for trapped particles at the SAA region, the steeper LET distribution is seen with very few high LET particles above $100 \mathrm{keV} / \mu \mathrm{m}$ as expected. Main component of these particles were assigned to be low energy $(\sim 10 \mathrm{MeV})$ protons by the spectroscopy of RRMD-I data. Note that in these distributions, the absolute intensity is somewhat ambiguous because the duration of the SAA passage is not strictly defined and a small contribution of some GCRs during the SAA passage might be included. In Fig. 6(b), the differential LET distribution of GCRs indicates a clear peak at the LET about $135 \mathrm{keV} / \mu \mathrm{m}$ for the GCR of Fe

(b)

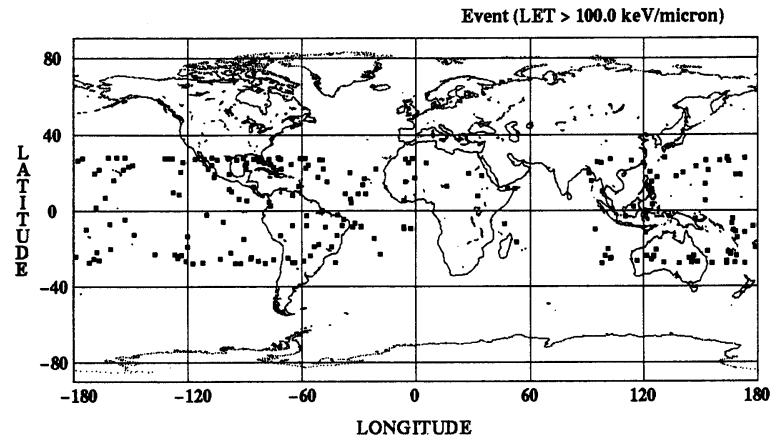

(c)

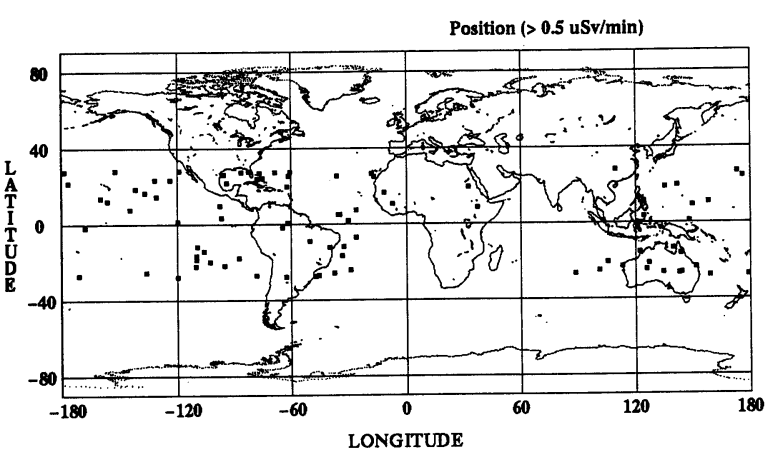

Fig. 5 (a) World-wide map of the Shuttle positions of every $2 \mathrm{~min}$ in which particle density for LET $>5 \mathrm{keV} / \mu \mathrm{m}$ is greater than 5 particles/min (closed squares) (IML-2). (b) Shuttle positions of incidence of particles of LET $>100 \mathrm{keV} / \mu \mathrm{m}$ (closed squares) (IML2). (c) Shuttle positions of high dose equivalent rate $>0.5 \mu \mathrm{Sv} / \mathrm{min}$ (closed squares) (IML-2). 
particles and several shoulders corresponding to the LET values of main abundant components of relativistic GCRs. So, we conclude here that the RRMD-I detector has responded as expected under the correct calibration.

\subsection{RRMD-II Results (S/MM\#4)}

The dosimetry by the RRMD-I telescope with two position detectors was the first attempt of real-time monitoring of space radiation achieved successfully for a continuous period of $251.3 \mathrm{~h}$ (IML-2) under the discrimination of LET $>5.0 \mathrm{keV} / \mu \mathrm{m}$. However, this telescope could not detect particles stopped in the PSD-2 from the bottom side entrance with the two position detector triggering, as mentioned above. The improved RRMD-II telescope with the third position detector at the bottom under the discrimination of LET $>3.5 \mathrm{keV} / \mu \mathrm{m}$ measured radiation for continuous $186.7 \mathrm{~h}\left(\mathrm{~S} / \mathrm{MM} \# 4: 51.6^{\circ}\right.$ x 300$400 \mathrm{~km}$ ). Then, the RRMD-II measurements confirmed to be negligibly small about $1.3 \%$ correction for particles stopping at PSD-2 from the bottom side (Sakaguchi et al., 1997) which were neglected in the IML-2 experiment to dose equivalent of the result of IML-2 $\left(28.5^{\circ} \times 300 \mathrm{~km}\right)$. At the altitude of $400 \mathrm{~km}$, this correction increases up to $2.1 \%$. However, only in the SAA region, this correction increases from $0.01 \%$ at $300 \mathrm{~km}$ to $3.0 \%$ at $400 \mathrm{~km}$. This implies that contribution for this kind of stopping particles becomes much more significant for higher altitude. So, the use of three position detector triggering system would be absolutely needed at $51.6^{\circ} \times 400 \mathrm{~km}$ (Doke et al., 1996). Also, the contribution of the SAA region to the total dose equivalent is about $15 \%$ (S/MM\#4: $51.6^{\circ} \times 300-400 \mathrm{~km}$ ) compared with less than $5 \%$ (IML-2: $28.5^{\circ} \times 300 \mathrm{~km}$ ) for LET $>3.5 \mathrm{keV} / \mu \mathrm{m}$.

In the S/MM\#4 mission, the Shuttle orbit data could be combined to the RRMD-II data in semi-real time. Then, we could construct the world-wide maps of count rates and dose equivalent rates at the orbit of $51.6^{\circ} \times 400 \mathrm{~km}$ in semireal time as shown in Figs. 7(a) and (b), respectively (Sakaguchi et al., 1997). In these figures, the big dense clusters are clearly seen at the SAA region (south-east of Brazil) and above Canada and south of Australia near geomagnetic poles as well as higher latitude regions. Comparing Fig. 7 with Fig. 5, it is seen that absorbed doses which are directly related with the particle density depend on the orbit altitude (SAA trapped particles contribution dominant) and dose equivalents on the orbit inclination (GCR contribution dominant).

In these two typical orbits of IML-2 (a small inclination $\mathrm{x}$ low altitude) and S/MM\#4 (a large inclination $\mathrm{x}$ high altitude), a comparison of the LET distributions are shown in Fig. 8. In this Figure, about 5 times larger intensities of S/MM\#4 than IML-2 are observed for this high LET region. This result at the high LET region will strongly reflects a effective quality factor $Q$ for all LET region above $0.2 \mathrm{keV} /$ $\mu \mathrm{m}$.

\subsection{TLD and CR-39 Results}

In the IML-2 experiment, dosimeter packages F1 and F4 were stored in the incubator of the "BIORACK" and in the rack of the "PEMBSIS" and packages F2 and F3 were attached to the side wall of RRMD "Detector Unit". And, all dosimeter packages (Dosimeter 1 - 4) of the S/MM\#4 experiment were attached around the wall of "Detector Unit" of RRMD-II. These dosimeter packages (FI - F4; Dosimeter 1 - 4) contain MSO-TLDs and CR-39 plastic track detectors which have a sensitivity threshold as low as LET of $3.9 \mathrm{keV} / \mu \mathrm{m}$ (Benton et al., 1986; Doke et al., 1995b), and LET distributions by CR-39 in all packages (a)

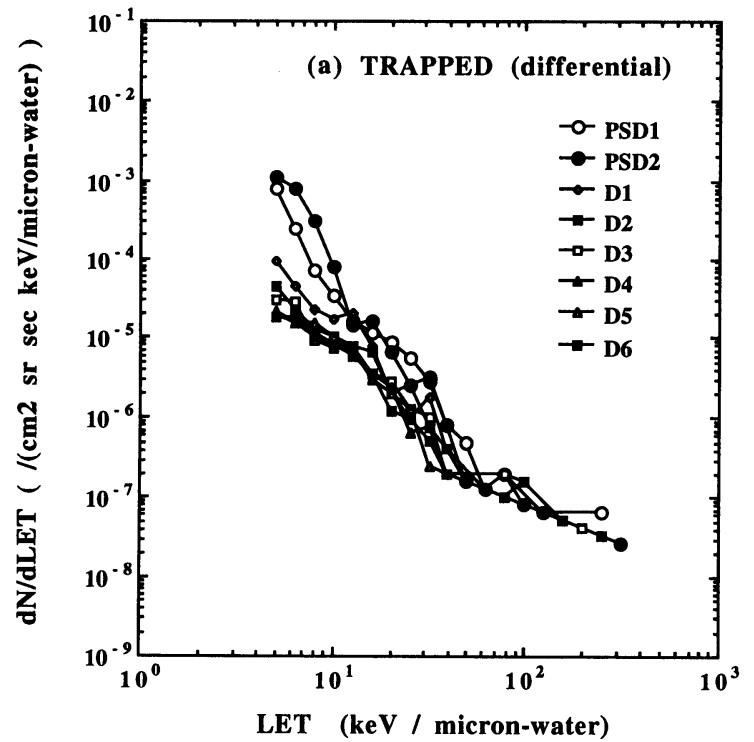

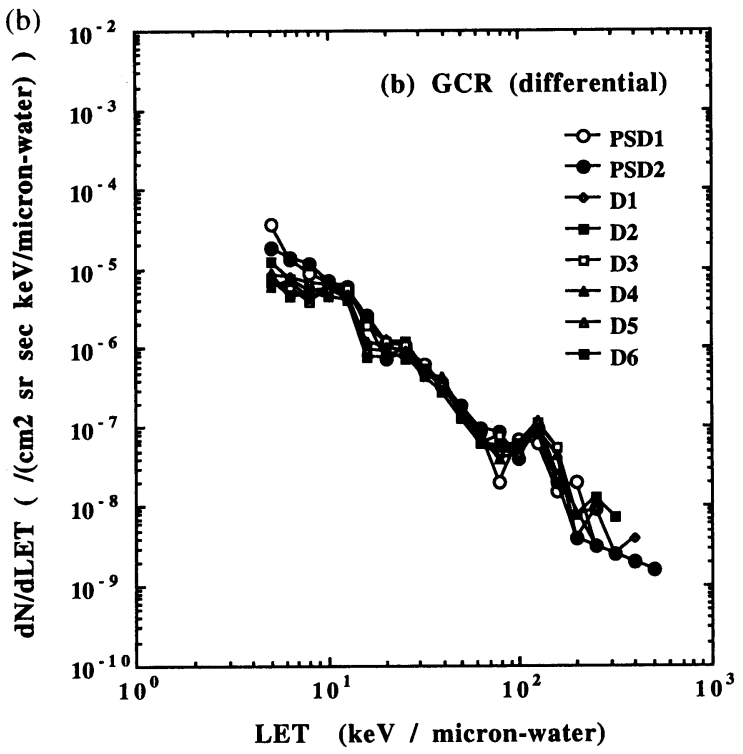

Fig. 6 (a) An example of differential LET distributions for trapped particles. Note that in these distributions,the absolute intensity is somewhat ambiguous because the duration of the SAA passage is not strictly defined and a small contribution of some GCRs during the SAA passage might be included (IML-2). (b) An example of differential LET distributions for GCRs (IML-2). 
(a)

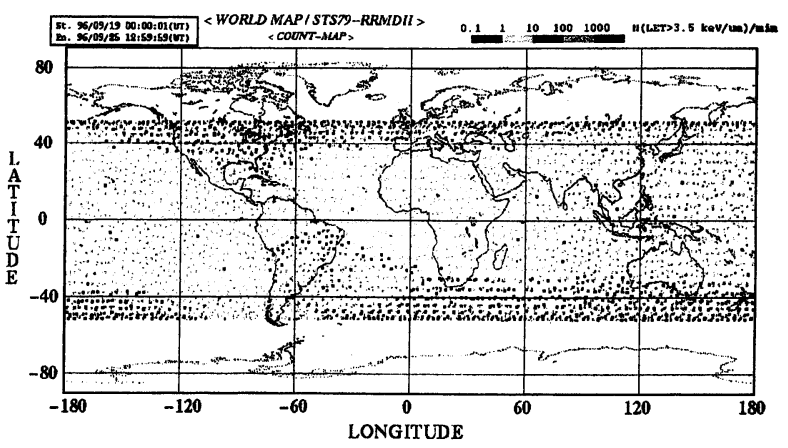

(b)

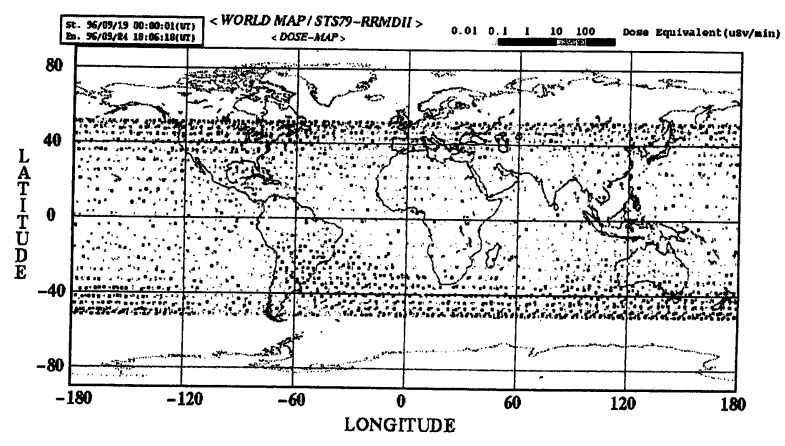

Fig. 7 (a) World-wide map of the Shuttle positions of count rate every 2 min for LET $>3.5 \mathrm{keV} / \mu \mathrm{m}$ (S/MM\#4)

(b) World-wide map of the Shuttle positions of dose equivalent rate every 2 minutes for LET> $3.5 \mathrm{keV} / \mu \mathrm{m}$ (S/MM\#4)

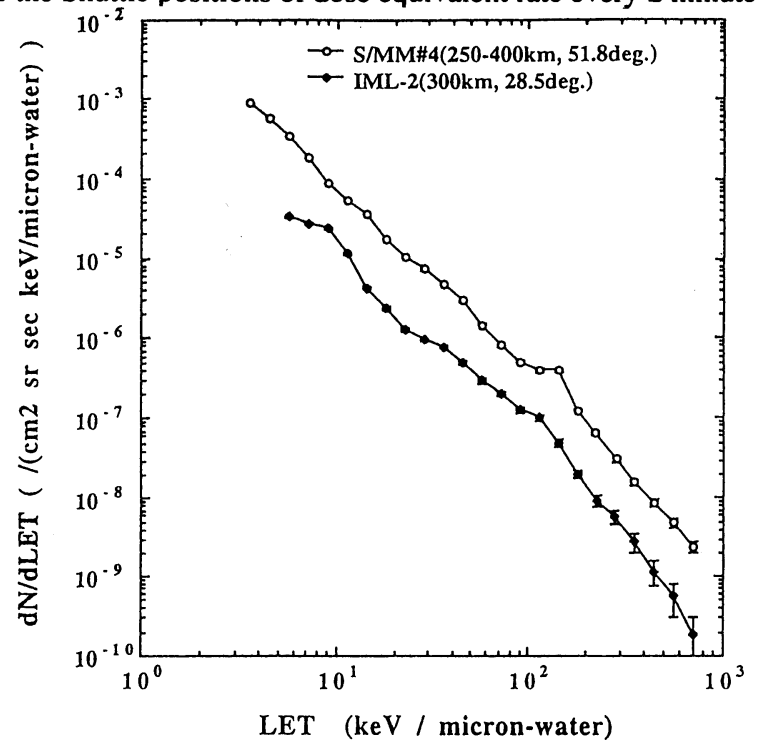

Fig. 8 A comparison of LET distributions between RRMD-I (IML-2) and RRMD-II (S/MM\#4).

(a)

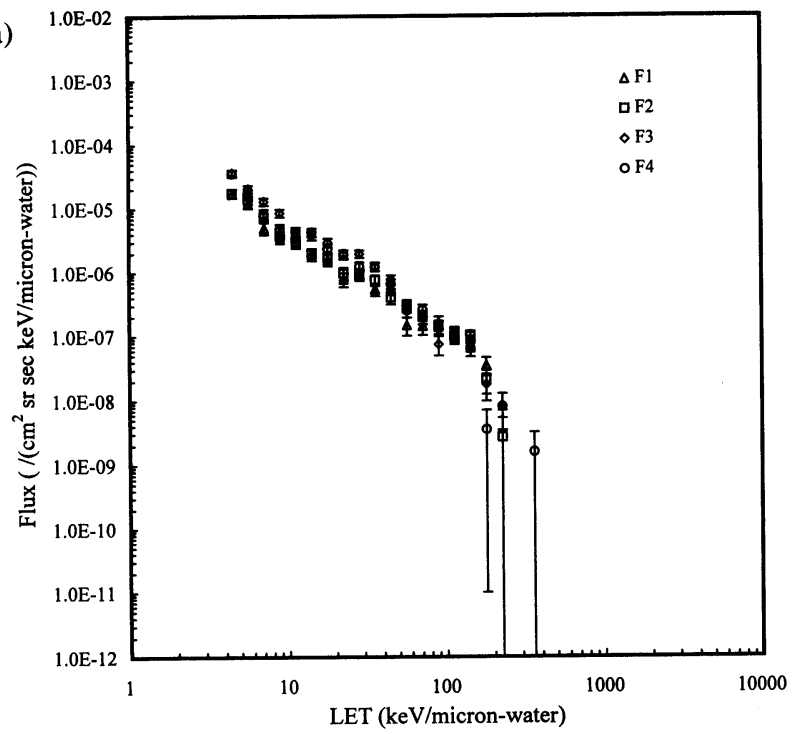

(b)

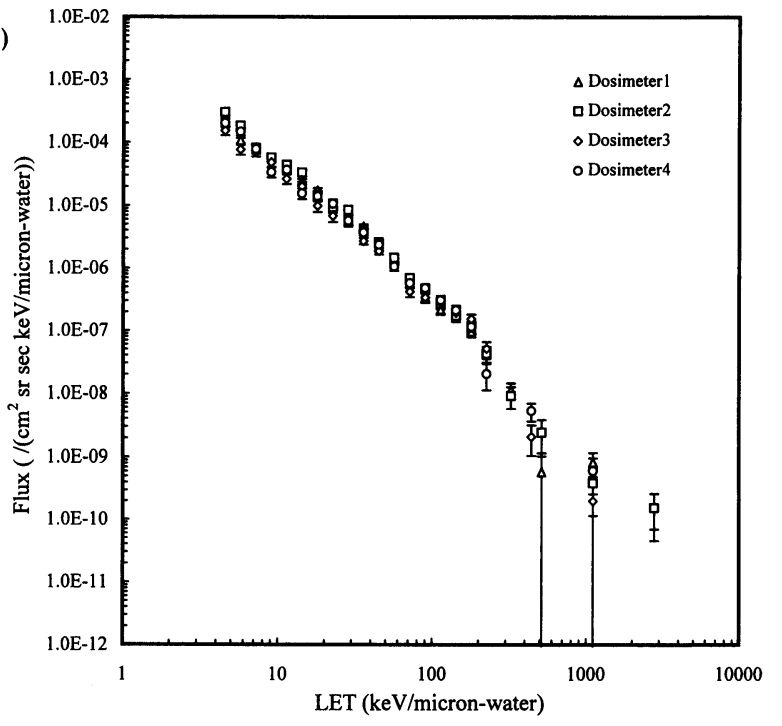

Fig. 9 (a) LET distributions by CR-39 in four dosimeter packages of IML-2. (b) LET distributions by CR-39 in four dosimeter packages of S/MM\#4. 

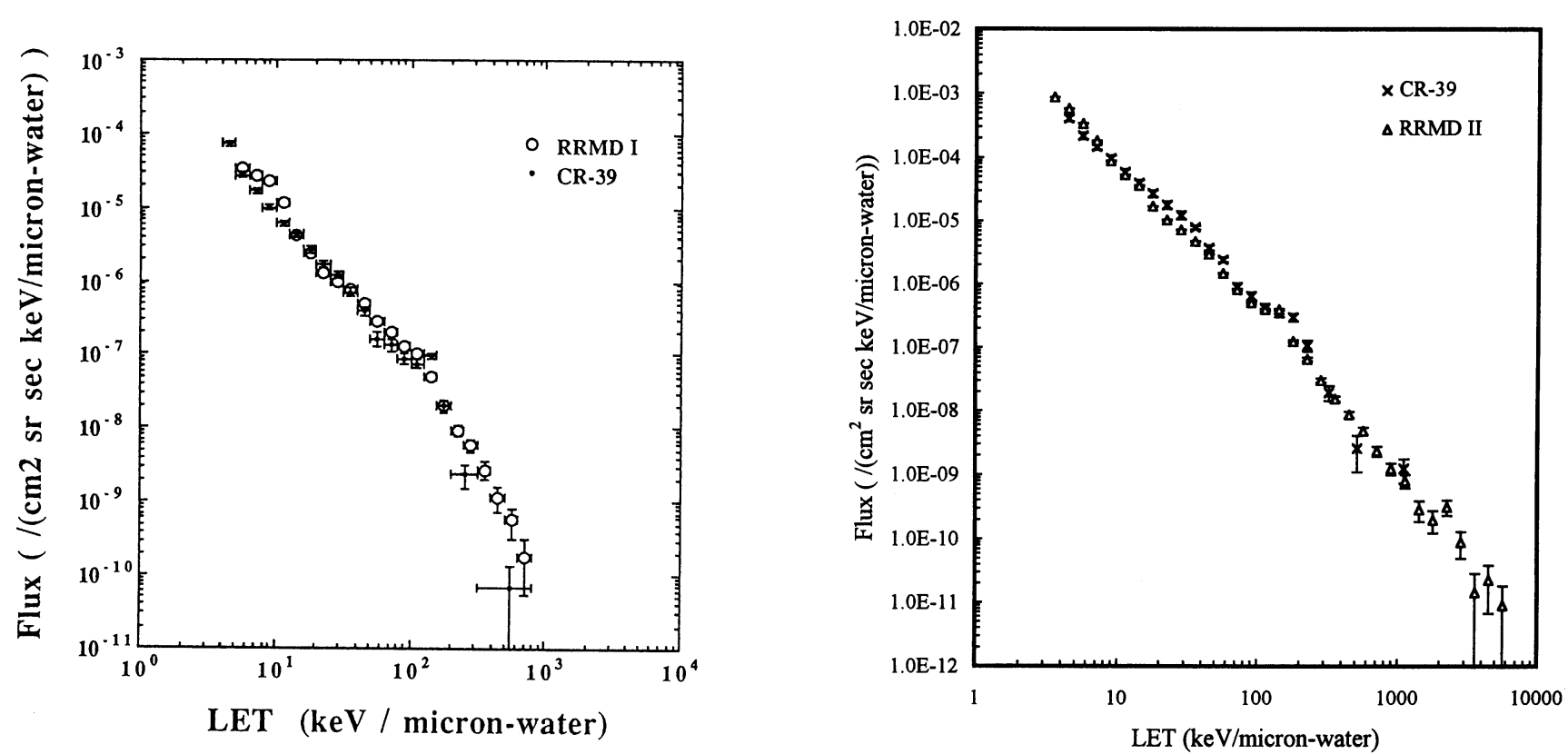

Fig. 10 (a) A comparison of differential LET distributions obtained by RRMD-I and CR-39 (IML-2).

(b) A comparison of differential LET distributions obtained by RRMD-II and CR-39 (S/MM\#4).

Table 1 (a) A summary of absorbed dose rates by TLDs(MSO-S) (D(TLD)), CR-39(HARZLAS) (D(CR)), total absorbed dose rates $(D($ all $))$, dose equivalent rates by CR-39 $(H(\mathrm{CR}))$, total dose equivalent rates $(H($ all $))$ and average quality factors $(Q F)$ for all LET region in the IML-2 experiment $\left(28.5^{\circ} \times 300 \mathrm{~km}: 14.68\right.$ days $)$.

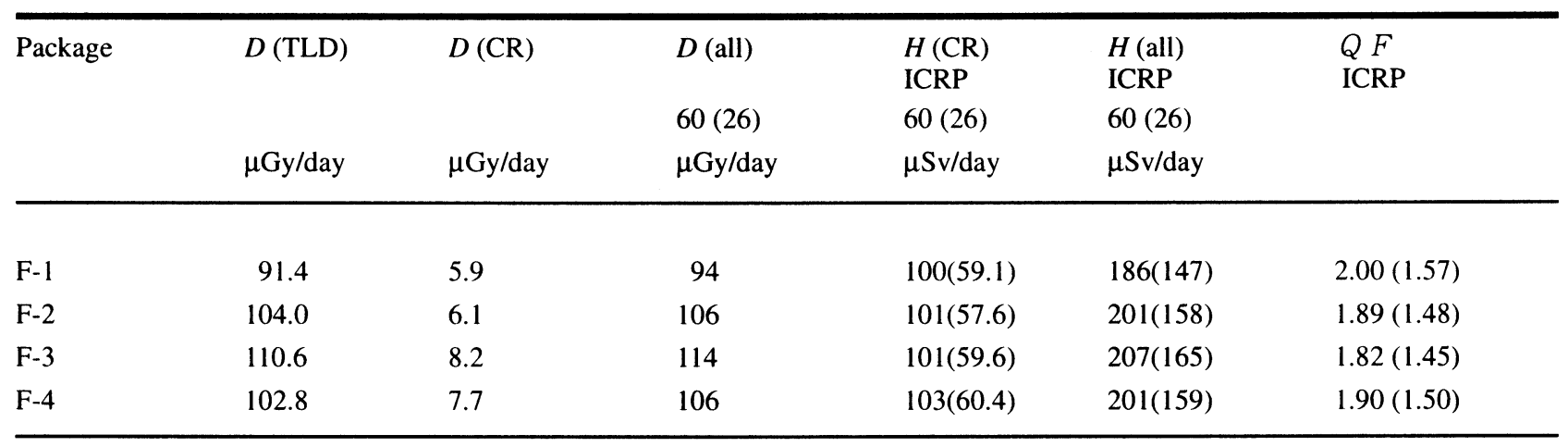

Table 1 (b) A summary of absorbed dose rates by TLDs (MSO-S) ( $D$ (TLD)), CR-39 (HARZLAS) ( $D(C R)$ ), total absorbed dose rates $(D($ all $))$, dose equivalent rates by CR-39 $(H(C R))$, total dose equivalent rates $(H($ all $))$ and average quality factors $(Q F)$ for all LET region in the $\mathrm{S} / \mathrm{MM} \# 4$ experiment $\left(51.6^{\circ}\right.$ x $400 \mathrm{~km}$ : 10.2 days $)$.

\begin{tabular}{|c|c|c|c|c|c|c|}
\hline Package & $\begin{array}{c}D \text { (TLD) } \\
\mu \mathrm{Gy} / \text { day }\end{array}$ & $\begin{array}{l}D(\mathrm{CR}) \\
\mu \mathrm{Gy} / \mathrm{day}\end{array}$ & $\begin{array}{l}D(\text { all }) \\
\mu G y / \text { day }\end{array}$ & $\begin{array}{l}H(\mathrm{CR}) \\
\text { ICRP } \\
60(26) \\
\mu \mathrm{Sv} / \text { day }\end{array}$ & $\begin{array}{l}H(\text { all }) \\
\text { ICRP } \\
60(26) \\
\mu S v / \text { day }\end{array}$ & $\begin{array}{l}Q F \\
\text { ICRP } \\
60(26)\end{array}$ \\
\hline Dosimeter 1 & 353.3 & 34.0 & 367 & $319(257)$ & $651(583)$ & $1.78(1.61)$ \\
\hline Dosimeter2 & 312.0 & 38.1 & 327 & $348(266)$ & $637(555)$ & $1.95(1.70)$ \\
\hline Dosimeter3 & 310.4 & 26.0 & 321 & $287(195)$ & $582(490)$ & $1.82(1.53)$ \\
\hline Dosimeter4 & 278.4 & 30.9 & 290 & $323(223)$ & $582(482)$ & $2.01(1.66)$ \\
\hline
\end{tabular}


were obtained by the measurements of major and minor axes of surface ellipse of etched tracks, and an amount of removal material, as shown in Figs. 9(a) for IML-2 and (b) for S/MM\#4. In Fig. 9(a), there seems to be some systematic difference that dosimeters $\mathrm{F} 1$ and $\mathrm{F} 4$ stored in the "BIORACK" and "PEMBSIS" show slightly lower intensities. However, there still remains the possibility within statistical and experimental errors. The distributions of all packages of S/MM\#4 are in very good agreement within statistical and experimental errors. In the comparison of Fig. 9 (a) and (b), in total, about 5 times larger intensities of S/MM\#4 than IML-2 with some LET dependence are also clearly seen for this high LET region. The results of MSO-TLDs reduced by the value of ground control ones are summarized in Table 1(a) and (b) for IML-2 and S/ MM\#4, respectively.

\subsection{Comparison of LET distributions by RRMDs and CR-39}

In Figs. 10(a) and (b), we compare the differential LET distributions by RRMD-I and CR-39 (IML-2), and RRMDII and CR-39 (S/MM\#4), respectively. The CR-39 data were obtained in the "Sample Holder" which was placed right above the RRMD telescopes. These distributions are in good agreement in the region of LET of $15-200 \mathrm{keV} / \mu \mathrm{m}$. The difference by a factor 4 - 5 above $200 \mathrm{keV} / \mu \mathrm{m}$ can be explained by the possibility that most of the slow heavy target fragments with extremely short range track in CR39 plate are less efficiently to be detected under the present etching condition of about $50 \mu \mathrm{m}$ removal layer, although the statistics of CR-39 data are very limited in this region. In the region of LET $<15 \mathrm{keV} / \mu \mathrm{m}$ especially in the IML2 case (Fig. 10 (a)), the surface ellipse of the CR-39 track become deformed at larger incident angles (Hayashi and Doke, 1980; Hayashi et al., 1996; Doke et al., 1997). Therefore, the LET value obtained by this deformed track would be estimated to be a value smaller than true one, resulting in lower intensity in this low LET region. The LET distributions of CR-39 were extrapolated down to a LET value of $3.5 \mathrm{keV} / \mu \mathrm{m}$, since the quality factor $Q$ is a function of LET (ICRP, 1977; ICRP, 1991). Thus, absorbed doses can be calculated by the integration of LET distribution and dose equivalents by the integration of LET distribution multiplied by quality factors (Doke et al., 1995 b) as mentioned in the RRMD case. So, we have corrected absorbed dose rate and dose equivalent rate of CR-39 by comparing the experimental results of RRMD-I and CR-39. Estimated correction factors are 1.18 for absorbed dose and 1.27 for dose equivalent at the region LET $>3.5 \mathrm{keV} / \mu \mathrm{m}$ in the case of the IML-2, where the CR-39 data have been obtained by the distribution extrapolated down to $3.5 \mathrm{keV} / \mu \mathrm{m}$ (Hayashi et al., 1996). These values have been applied to the evaluation of absorbed dose rate and dose equivalent rate in the IML-2 dosimeter packages (FI - F4). For the CR-39 of S/MM\#4, this correction factors seem to be smaller by seeing "face" of etched tracks and now are under analysis. So, at the present stage, the correction factor 1.0 have been applied for both of absorbed dose and dose equivalent. These values have been applied to Tables 1(a) for IML-2 and (b) for S/ $\mathrm{MM \# 4}$. In these tables, MSO-TLDs results give absorbed doses for all natural LET values $>0.2 \mathrm{keV} / \mu \mathrm{m}$ and CR-39 results give absorbed doses and dose equivalents for LET $>3.5 \mathrm{keV} / \mu \mathrm{m}$ by the LET distribution. From this combination of data of MSO-TLDs and CR-39, we estimated total absorbed doses and dose equivalents for the whole LET range as mentioned above (Doke $e t$ al., 1995b; Hayashi et al., 1996). These results and average quality factors are also shown in Tables 1(a) and (b).

\section{Summary and Conclusion}

The first attempt of real-time mesurements of LET distribution and radiation dosimetry using the RRMD-I have been achieved successfully. The LET distributions by the RRMDs and CR-39 in the LET region of 15 - 200 $\mathrm{keV} / \mu \mathrm{m}$ are in very good agreement. In the region above $200 \mathrm{keV} / \mu \mathrm{m}$ and below $15 \mathrm{keV} / \mu \mathrm{m}$, the difference between the two distributions can be explained by the characteristics of CR-39 track detectors.

Table 1(a) and (b) show summaries of the results by MSO-TLDs and CR-39 for all natural LET values $>0.2$ $\mathrm{keV} / \mu \mathrm{m}$, where the real exposure times were assumed as 14.68 days (IML-2) and 10.2 days (S/MM\#4). For the whole LET range ( $>0.2 \mathrm{keV} / \mu \mathrm{m})$ obtained by TLDs and CR39 in these two typical orbits (a small inclination $x$ low altitude) and (a large inclination $\mathrm{x}$ high altitude), absorbed dose rates range from 94 to $114 \mu \mathrm{Gy} /$ day, dose equivalent rates from 186 to $207 \mu \mathrm{Sv} /$ day and average quality factors from 1.82 to 2.00 depending on the locations and directions of detectors inside the Spacelab at the highly protected IML- 2 orbit $\left(28.5^{\circ} \times 300 \mathrm{~km}\right)$, and also, absorbed dose rates range from 290 to $367 \mu \mathrm{Gy} /$ day, dose equivalent rates from 582 to $651 \mu \mathrm{Sv} /$ day and average quality factors from 1.78 to 2.01 depending on the dosimeter packages around the RRMD-II detector at the S/MM\#4 orbit $\left(51.6^{\circ} \times 400\right.$ $\mathrm{km})$. These results are consistent with results obtained by others with the same orbit parameters (for example Reitz et al., 1996).

The result at the high LET region will strongly reflect a effective quality factor for all LET region above $0.2 \mathrm{keV} /$ $\mu \mathrm{m}$. In general, it is seen that absorbed doses depend on the orbit altitude (SAA trapped particles contribution dominant) and dose equivalents on the orbit inclination (GCR contribution dominant).

The CR-39 data generate much more correct LET distribution if the deformation effect of larger incident angles for used CR-39 is calibrated well. Also, the present RRMD-II could not detect particles of LET $<3.5 \mathrm{keV} / \mu \mathrm{m}$. In May, 1997, the modified RRMD detector which can observe minimum ionizing particles with LET $=0.2 \mathrm{keV} /$ $\mu \mathrm{m}$, will be flown in STS-84 (S/MM\#6) (Doke, 1994b; Doke et al., 1996) and then various applications of this detector type to real-time space dosimetry will be considered. 


\section{Acknowledgment}

We would like to thank the accelerator teams of the RIKEN Ring Cyclotron, the TIARA Cyclotron of JAERI, Takasaki and the HIMAC of the National Institute for Radiological Science for the kind and careful support in the beam exposures. We would also like to thank the JSC ODRC team for great support to obtain the orbit data. The results were obtained from the joint RRMD program of NASA and NASDA.

\section{References}

Badhwar G. D., Braby L. A., Cucinotta F. A. and Atwell W. (1992), Nucl. Tracks Radiat. Meas. 20, 447.

Badhwar G. D., Cucinotta F. A., Braby L. A. and Konradi A. (1994), Radiat. Meas. 139, 344.

Badhwar G. D., Konradi A., Atwell., Golightly M. J., Cutinotta F. A., Willson J. W., Petrov V. M., Tchernykh I. V., Shurshakov V. A. and Lobokov A. P. (1996), Radiat. Meas. 26, 147.

Benton E. V. (1968), Report USNRDL-TR-68-14, US Naval Radiological Defense Laboratory, San Francisco, U.S.A.

Benton E. V., Ogura K., Frank A. L., Atallah T. M. and Rowe V. (1986), Nucl. Tracks Radiat. Meas. 12, 79.

Doke T. (1994a), Technical Report No.94-4, Advanced Research Center for Science and Engineering, Waseda University.

Doke T.(1994b), Technical Report No.94-31, Advanced Research Center for Science and Engineering, Waseda University.

Doke T., et al. (1994), J. Geomag. \& Geoelectr. 46, 713.

Doke T., Hayashi T., Kikuchi J., Hasebe N., Nagaoka S., Kato M. and Badhwar G. D. (1995a), Nucl. Instr. Meth. in Phys. Res. A365, 524.

Doke T., Hayashi T., Nagaoka S., Ogura K. and Takeuchi R. (1995b), Radiat. Meas. 24, 75.

Doke T., Hayashi T., Hasebe N., Kikuchi J., Kono S., Murakami T., Sakaguchi T., Takahashi K. and Takashima T. (1996), Jpn. J. Appl. Phys. 35, 6241.

Doke T., Hayashi T., Kobayashi M. and Watanabe A. (1997), Radiat. Meas., 28, 445.

Golightly M.J., Hardy K. and Ouam W. (1994), Radiat. Meas. 23,25 .

Hayashi T. and Doke T. (1980), Nucl. Instr. \& Meth. 174, 349.

Hayashi T., Doke T., Hasebe N. and Fujiki K. (1994), Jpn. J. Appl. Phys. 33, 4106.

Hayashi T., Doke T., Kikuchi J., Takeuchi R., Hasebe N., Ogura K.,Nagaoka S., Kato M. and Badhwar G. D. (1996), Radiat. Meas. 26, 935.

ICRP (1977) Publication 26, Annals of the ICRP I (3). Pergamon Press, Oxford.

ICRP (1991) Publication 60, Annals of the ICRP 21. Pergamon Press, Oxford.

Nagaoka S., Doke T., Hayashi T., Ogura K., Yamada H., Takahashi T., Yatagai F., Yato O., Ishikawa M., Takashima F., Tanaoka H. and Harada K. (1993), Summary Report on the Science Results of Fuwatto's 92 Space Experiment. NASA, 140.

Reitz G., Beaujean R., Heilmann C., Kopp J., Leicher M. and Strauch K. (1996), Radiat. Meas. 26, 979.
Sakaguchi T., Doke T., Hayashi T., Kikuchi J., Hasebe N., Kashiwagi T., Takashima T., Takahashi K., Nakano T., Nagaoka S., Takahashi S., Yamanaka H., Yamaguchi K. and Badhwar G.D. (1997) Jpn. J. Appl. Phys. 36, 7453. 